\title{
A Numerical Framework for the Analysis of Handoff Delay Component in Proxy NEMO Environment
}

\author{
Shayla Islam ${ }^{1}$, Aisha-Hassan A. Hashim ${ }^{1}$, Azween Abdullah ${ }^{2}$ \\ and Mohammad Kamrul Hasan ${ }^{1}$ \\ ${ }^{1}$ Faculty of Engineering, International Islamic University Malaysia, Jalan \\ Gombak 53100, Kuala Lumpur, Malaysia \\ ${ }^{2}$ SOCIT, Taylor's University, Jalan Taylors, Subang Jaya 47500, Selangor, \\ Malaysia \\ iium19612@hotmail.com
}

\begin{abstract}
Network Mobility Basic Support Protocol (NEMO-BSP), the existing IETF standard for mobile network support, signifies an important portion for future heterogeneous wireless access networks. The reason is to provide continuous Internet connectivity during movement of Mobile Router (MR) in NEMO. This paper conducted a quantitative analysis on the handoff delay component of NEMO-BSP as well as its existing enhancements, i.e., Fast NEMO (F-NEMO) handoffs, and an Extension of F-NEMO (EF$N E M O)$, using the numerical framework. The mathematical scenario includes two access routers, one local home agent and up to 20 MRs that interrelate by two different wireless access networks are mainly WiFi and WiMAX. The analysis offers quantitative outcomes of the performance enhancements achieved via the proposed improvements concerning handoff delay gain, packet loss, and packet loss ratio. The numerical results assist in understanding the influence of link switching delay, mobility rate, and radius on the handoff delay gain, packet loss, and packet loss ratio. The results of this analysis will also be aided to pick an appropriate mobility management scheme for Proxy NEMO environment.
\end{abstract}

Keywords: NEMO-BSP; F-NEMO; heterogeneous wireless access networks; handoff delay component.

\section{Introduction}

Next Generation Wireless Access Networks (NGWANs) are enduring a substantial improvement in terms of the amount of mobile user as well as the amount of applications assisted. Those procedures will provide to the mobile users the chance to travel among different wireless access technologies to support inter technology handoff. Therefore, the Network Mobility Basic Support Protocol (NEMO-BSP) has been standardized by the NEMO Working Group (NEMO WG) as the basic protocols to support continuous Internet connectivity during handoff in mobile networks [1-2]. Several developments of the NEMO-BSP have already been proposed. The reason is that, in certain circumstances NEMO-BSP could effect in a poor handoff performance. During handoff of Mobile Router (MR) from one subnet to another in NEMO environment, the single-interfaced MR need to change its primary Care of Address (CoA) recurrently. This increases single point of failure probability that results in higher handoff delay and packet loss [3-8]. Hence, schemes integrating Fast MIPv6 concept with NEMO (F-NEMO) have developed to improve handoff performance in NEMO [7]. However, F-NEMO experiences a higher tunneling during movement among different access technologies. 
The main aim of this paper is to investigate the effect of different parameters on the handoff performance as endured by a single-interfaced MR in mobile network as well as compares the performance achieved by the existing enhancements (i.e., F-NEMO, or proposed Extension of F-NEMO) with the standard NEMO-BSP.

The major contributions of this paper is sketched as follows: (i) proposed an Extension of F-NEMO (EF-NEMO) (ii) evaluate as well as compare the numerical outcomes of NEMO-BSP, F-NEMO and proposed EF-NEMO using numerical framework in terms of handoff delay gain, packet loss and packet loss ratio.

The remaining portion of this paper is organized as follows: Section 2 provides a synopsis of NEMO BSP enhancement are mainly F-NEMO which is followed by the proposed EF-NEMO scheme in Section 3. A numerical framework is developed to assess as well as compare the numerical results of NEMO-BSP, F-NEMO and proposed EFNEMO in Section 4. Finally, ending remarks are offered in Section 5.

\section{Overview of Fast Handoff Scheme in NEMO (F-NEMO)}

In order to improve the handoff performance of NEMO-BSP, Fast MIPv6 (FMIPv6) architecture has integrated with NEMO-BSP which can be named as Fast NEMO (FNEMO) [9]. In the handover process of FNEMO, the MR joins in the handoff in support of Mobile Network Nodes (MNNs). The foremost hypothesis behind F-NEMO is to execute several portions of the handoff procedure in advance. Likewise, a bi-directional tunnel among the Previous Access Router (PAR) and the New Access Router (NAR) is created in this technique. Buffering mechanism is also applied in this process. Therefore, F-NEMO can reduce packet loss as well as improve the handoff performance. However, Duplication Address Detection (DAD) mechanism need to be performed in F-NEMO scheme to verify whether the newly created CoA is valid or not. This leads to increase higher handoff delay, packet loss as well as degrade network performance. The basic handoff scenario of F-NEMO scheme is illustrated in Figure 1.

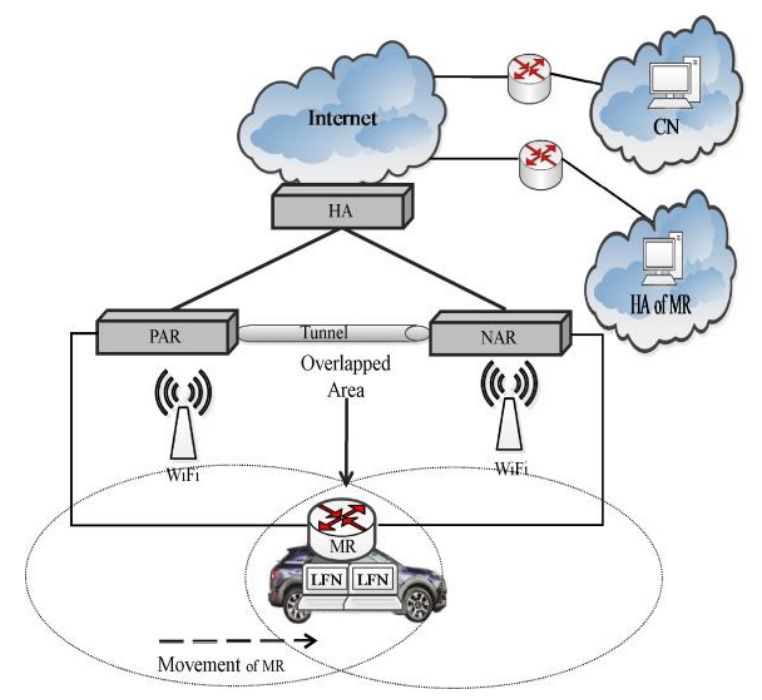

Figure 1. Basic Handoff Scenario of F-NEMO Scheme

\section{Proposed Extension of the FNEMO Scheme}

An Extension of F-NEMO (EF-NEMO) scheme is proposed in Proxy NEMO environment. In EF-NEMO, each mobility related Layer 3 (L3) signaling is handled by the network entities are mainly Mobile Access Gateway (MAG) as well as Local Mobility Anchor (LMA). 
The LMA is responsible for preserving the accessibility state of the MR as the Home Agent (HA) does in NEMO-BSP and F-NEMO. In contrast, The MAG is responsible to achieve mobility management for the MR in PNEMO environment. Thus, it is possible to improve handoff performance without managing any mobility related L3 signaling message via the MR or MNN in the EF-NEMO. According to the proposed scheme, MR initially attached with the MAG1 (i.e., WiFi interface). During movement from MAG 2 to MAG 3 (i.e., WiFi to WiMAX), binding update registration messages is encapsulated by exchanging Handoff Initiations (HI) and Handoff Acknowledgement (HAck) message in advance among MAG and LMA. In consequence, a temporary tunnel is created among MAG3 and LMA. Thus, it is probable to ease tunneling overhead. This leads to improve network performance as well as reduce handoff delay during handoff of MR among different access technologies. It is considered that, MR supports Logical Interface (LI) in order to use multiple interfaces simultaneously during handoff. The handoff scenario of the proposed EF-NEMO scheme is shown in Figure 2.

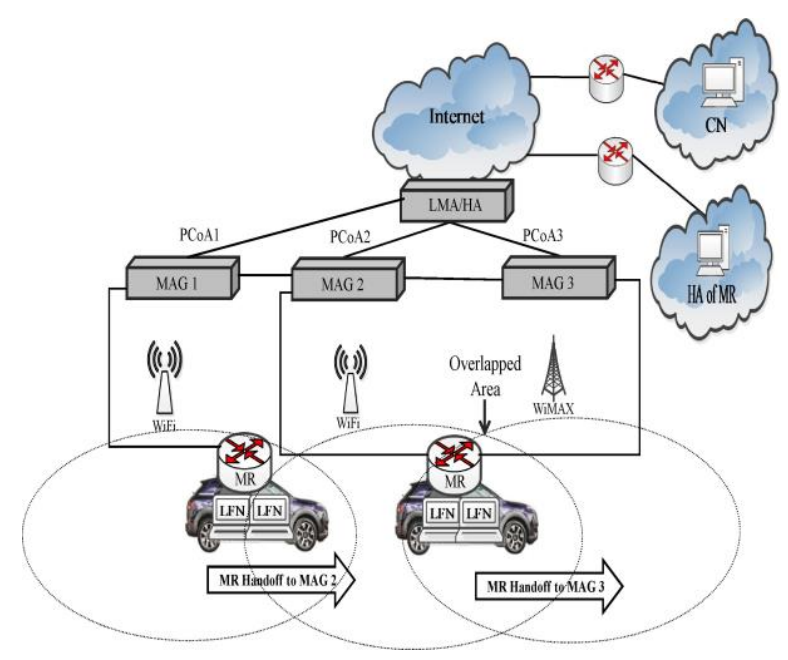

Figure 2. Handoff Scenario of the proposed EF-NEMO Scheme

\section{Performance Evaluation}

This section develop a numerical framework as depicts in Figure 3 to compare the handoff performance of NEMO-BSP, F-NEMO and EF-NEMO. In Figure 3, MAG1 and MAG2 signify Access Routers (AR) as PAR and NAR in F-NEMO whereas LMA works as local HA as HA in NEMO-BSP and F-NEMO [9-11].

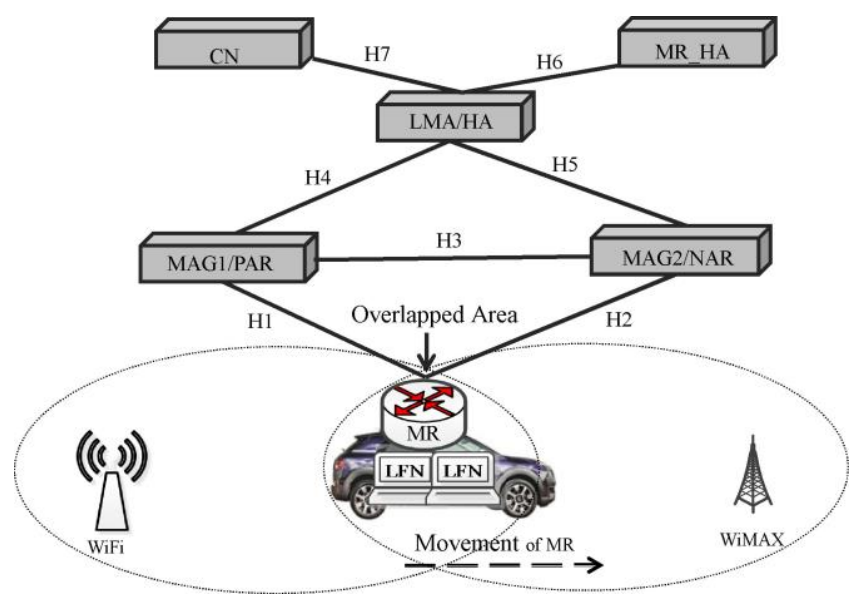

Figure 3. Numerical Framework of the proposed EF-NEMO Scheme 


\subsection{Handoff Delay Components Analysis}

A MR is not capable to receive any data packets on its new access network until finishes the handoff process. The handoff delay is influenced via several components such as Link Switching Delay $\left(\mathrm{D}_{\mathrm{LS}}\right)$, Movement Detection Delay $\left(\mathrm{D}_{\mathrm{MD}}\right)$, Duplicate Address Detection Delay $\left(\mathrm{D}_{\mathrm{DAD}}\right)$ and Registration Delay $\left(\mathrm{D}_{\mathrm{R}}\right)$.

The handoff delay for NEMO-BSP, F-NEMO and proposed EF-NEMO can numerically be expressed as:

$$
\begin{gathered}
D_{H D}^{N E M O-B S P}=D_{L S}+D_{M D}+D_{D A D}+D_{R} \\
D_{H D}^{F-N E M O}=\left(1-p_{p f}\right) \times P D_{H D}^{F-N E M O} \\
D_{H D}^{E F-N E M O}=T_{L 2}+\max \left(P D_{H D}^{E F-N E M O}\right)
\end{gathered}
$$

The Partial Delay for F-NEMO and proposed EF-NEMO can be symbolized as $P D_{H D}^{F-N E M O}$ and $P D_{H D}^{E F-N E M O}$ respectively. Thus, $P D_{H D}^{F-N E M O}$ and $P D_{H D}^{E F-N E M O}$ is further broken down to:

$$
\begin{aligned}
& P D_{H L}^{F-N E M O}=D_{F A S T}+D_{L 2}+D_{N E W}+D_{R E G}
\end{aligned}
$$

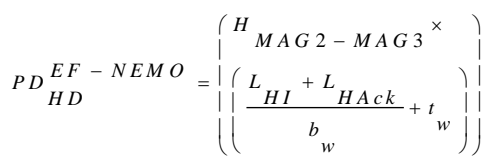

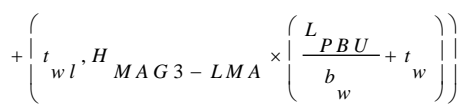

In Equation 4 and 5, $\mathrm{H}$ represents the hop distance whereas $\mathrm{L}$ denotes length of signaling message. Wired link delay as well as wireless link delay is denoted as $t_{w l}$ and $T_{w}$ respectively.

Therefore, the comparative Handoff Delay Gain (HDG) of EF-NEMO can be expressed as:

$$
\begin{gathered}
G_{H D}^{N E M O-B S P / E F-N E M O}=\frac{D_{H L}^{N E M O-B S P}}{T_{H D}^{E F-N E M O}} \\
G_{H D}^{F-N E M O / E F-N E M O}=\frac{D_{H D}^{F-N E M O}}{D_{H D}^{E F-N E M O}}
\end{gathered}
$$

Consequently, the Packet Loss Ratio (PLR) of the NEMO-BSP, F-NEMO and EFNEMO can be calculated as follows:

$$
\begin{aligned}
L_{P L R}^{N E M O-B S P} & =\frac{L_{\text {Loss }}^{N E M O-B S P}}{T_{\text {cell }}} \times 100 \% \\
L_{P L R}^{F-N E M O} & =\frac{L_{\text {Loss }}^{F-N E O}}{T_{\text {cell }}} \times 100 \% \\
L_{P L R}^{E F-N E M O} & =\frac{L_{\text {Loss }}^{E F-N E M O}}{T_{\text {cell }}} \times 100 \%
\end{aligned}
$$




$$
\text { where, } T_{\text {cell }}=\frac{2 r}{v}
$$

Here, radius of a cell and speed of MR can be symbolized as $r$ and $v$ respectively. Moreover, packet loss for NEMO-BSP, F-NEMO and EF-NEMO can be denotes as $L_{\text {Loss }}^{\text {NEMO-BSP }}, L_{\text {Loss }}^{F-N E M O}$ and $L_{\text {Loss }}^{E F-N E M O}$ respectively. So, $L_{\text {Loss }}^{N E M O-B S P}, L_{\text {Loss }}^{F-N E M O}$ and $L_{L o s s}^{E F-N E M O}$ can be expressed as:

$$
\begin{aligned}
& L_{\text {Loss }}^{N E M O-B S P}=\lambda_{p} \times \mu_{h} \times N_{M R} \times\left(D_{H D}^{N E M O-B S P}\right) \\
& L_{\text {Loss }}^{F-N E M O}=\lambda_{p} \times \mu_{h} \times N_{M R} \times\left(D_{H D}^{F-N E M O}\right) \\
& L_{\text {Loss }}^{E F-N E M O}=\lambda_{p} \times \mu_{h} \times N_{M R} \times\left\{\begin{array}{l}
\left.T_{L S}+H_{M A G-M A G} \times\right) \\
\left.\left\{\left(\frac{L_{H I}}{b_{w d}}+t_{w}\right)\right\}\right) \\
\left\{\left(\frac{L_{\text {HAck }}}{b_{w d}}+t_{w}\right)\right\}
\end{array}\right\}
\end{aligned}
$$

\subsection{Result Analysis}

The relative numerical outcomes of the proposed EF-NEMO are demonstrated in this section. It quantitatively compares the proposed approach with NEMO-BSP as well as FNEMO regarding the comparative handoff delay gain, packet loss and packet loss ratio with the aim of analyzing the enhancements as well as limitations. Table 1 specifies the parameter values used for numerical evaluation [9-11]

\section{Table 1. Parameters used for Quantitative Analysis [9-11]}

\begin{tabular}{|l|l|}
\hline Parameters & Values \\
\hline $\mathrm{T}_{\mathrm{ls}}$ & {$[50-300] \mathrm{ms}$} \\
\hline $\mathrm{P}_{\mathrm{wlf}}$ & 0.5 \\
\hline $\mathrm{B}_{\mathrm{wl}}$ & $54 \mathrm{mb} / \mathrm{s}$ \\
\hline $\mathrm{B}_{\mathrm{wd}}$ & $1000 \mathrm{mb} / \mathrm{s}$ \\
\hline $\mathrm{t}_{\mathrm{wl}}$ & $2 \mathrm{~ms}$ \\
\hline $\mathrm{t}_{\mathrm{wd}}$ & $0.5 \mathrm{~ms}$ \\
\hline Length of BU, PBU, FBU message & 52 bytes \\
\hline Length of HI, RS message & 52 bytes \\
\hline Length of RA message & 92 bytes \\
\hline Hop distance, $(\mathrm{a}=\mathrm{b}=\mathrm{c})$ & 1 \\
\hline Hop distance, $(\mathrm{d}=\mathrm{e})$ & 5 \\
\hline
\end{tabular}

Figure 4 illustrates the relative handoff delay gain against link switching delay $\left(D_{L S}\right)$ with varying $\tau$. According to Figure 4 , changing the value of $D_{L S}$ does not have obvious effect on the comparative handoff delay gain of the proposed EF-NEMO and F-NEMO. This is due to separating registration procedure from the acute handoff process in the proposed approach. In contrast, for NEMO-BSP when MR changes among different access technologies (i.e. WiFi to WiMAX), it is essential to do the registration with its home network for each frequent movement. This ultimately increase the handoff delay and degrade the network performance. However, the relative handoff gain of F-NEMO 
eases as $\tau$ rises, whereas that of EF-NEMO remains approximately the same, nonetheless of $\tau$. This is because of increasing tunneling overhead of F-NEMO via wireless link. Besides, the proposed approach supports LI on PNEMO environment. Thus, it is capable to use different access technologies at the overlapping area in PNEMO network. This leads to improve handoff performance.

According to Figure 5, it is observed that the packet loss escalates as soon as increase the mobility rate of MR for each scheme. However, the performance of EF-NEMO is significantly enhanced compared to NEMO-BSP and F-NEMO. The reason is that, EFNEMO eradicates the cost for tunneling during frequent movement between different access technologies. Moreover, increase mobility rate results in shorter residence time and thus recurrent handoffs.

Figure 6 depicts the ratio of packet loss for EF-NEMO and compared it with NEMOBSP as well as F-NEMO respectively. The mobility rate of MR is kept constant with varying the radius of cell from 0 to 300 meter as appeared in Figure 6. The loss ratio for packet loss fluctuates in between 0 to $3.5 \%$. In contrast, EF-NEMO shows minor loss ratio because of using multiple interfaces concurrently during handoff compared to singleinterfaced MR (i.e. NEMO BSP as well as F-NEMO).

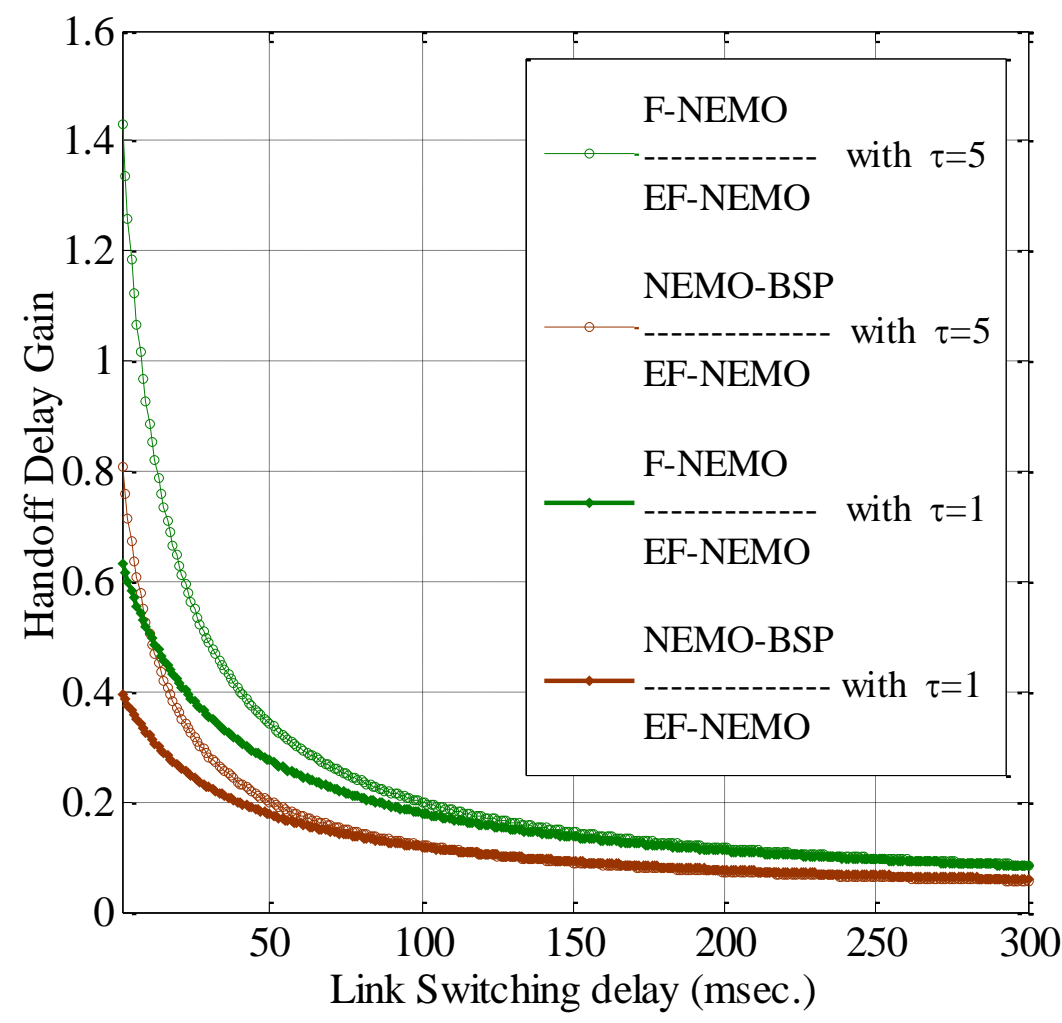

Figure 4. Handoff Delay Gain vs. Link Switching Delay 


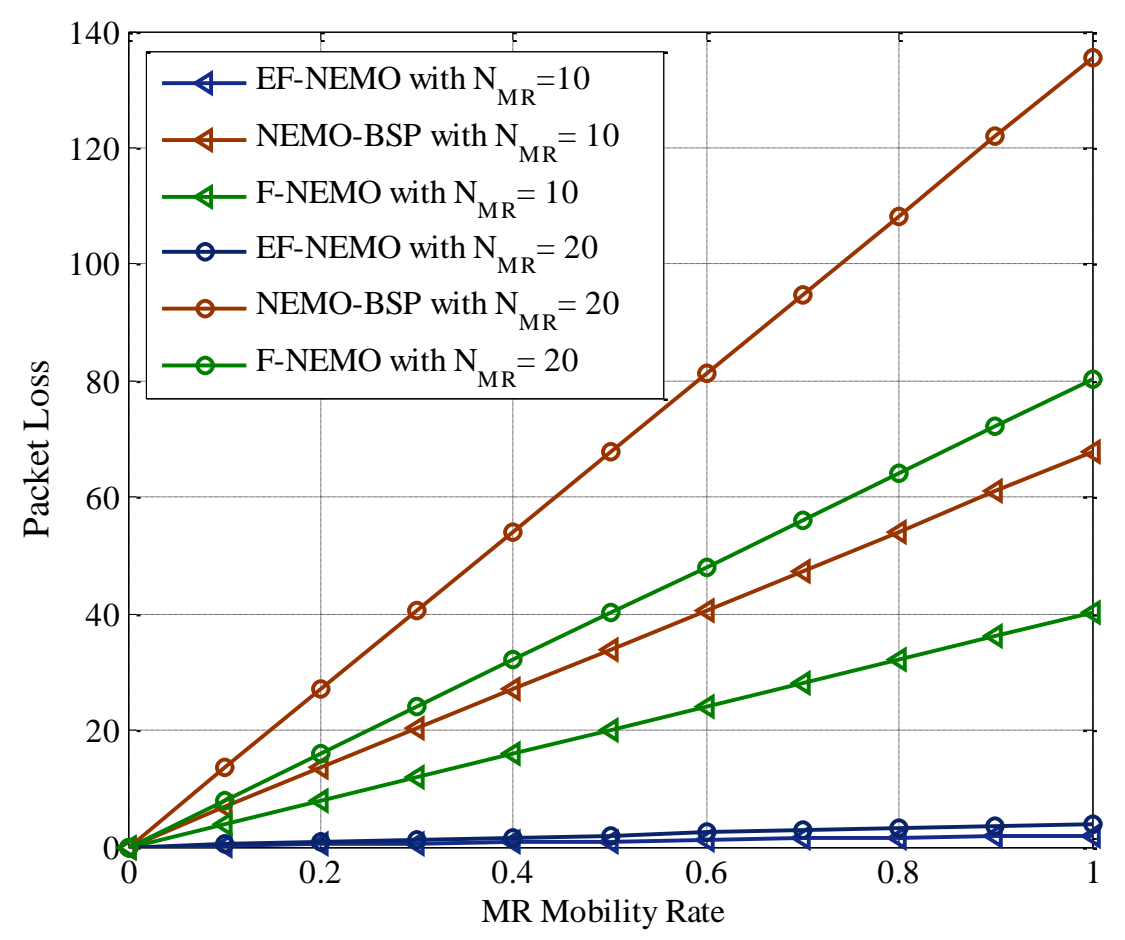

Figure 5. Packet Loss vs. MR Mobility Rate

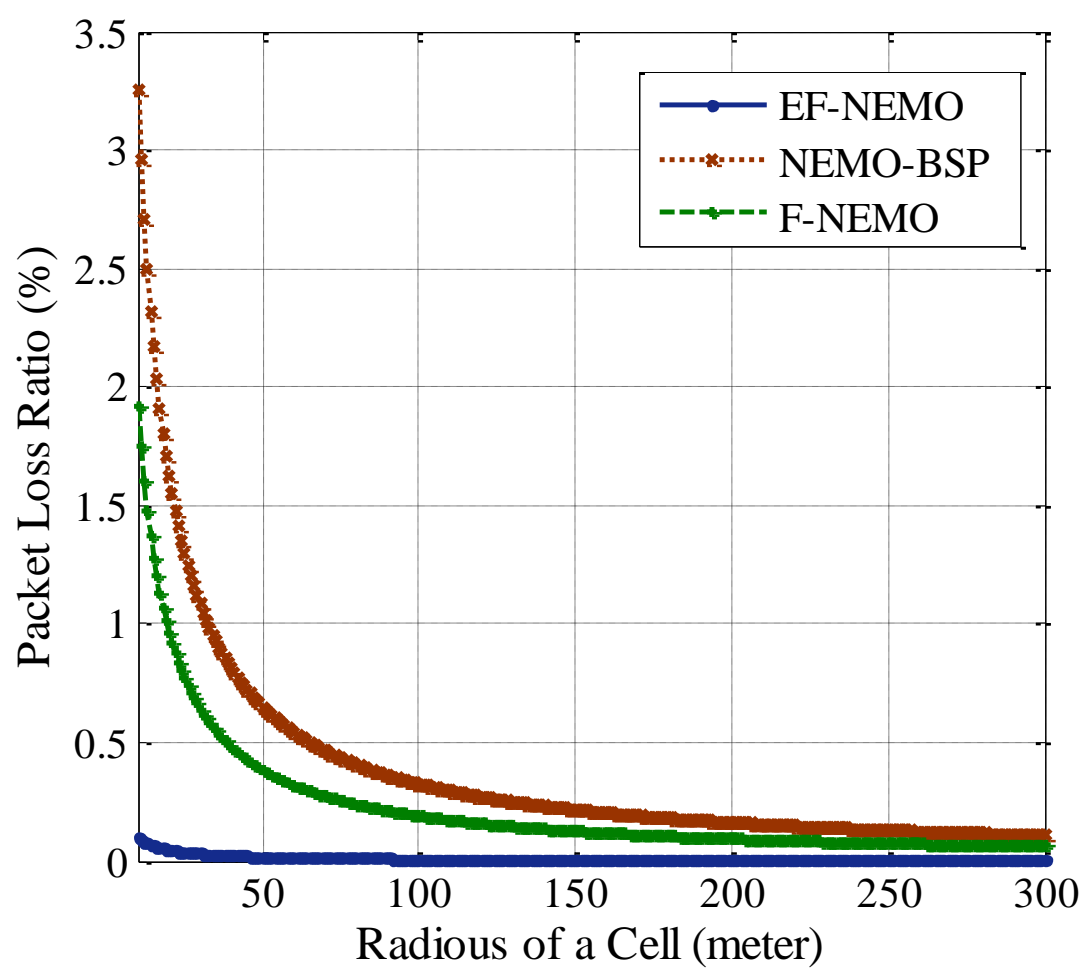

Figure 6. Packet Loss Ratio vs. Radius of a Cell 


\section{Conclusion}

This paper presented the performance of mobility management schemes in Proxy NEMO (PNEMO) environment during inter technology handoff. We evaluated the performance of three schemes are mainly NEMO-BSP, F-NEMO and EF-NEMO by using a numerical framework. Handoff delay, packet loss, and packet loss ratio have been considered as performance metrics. Our findings indicate that, for usual network configuration as well as system parameters, EF-NEMO has reduced handoff delay, packet loss and packet loss ratio. The reason is that, the MR in the EF-NEMO is capable to use multiple interfaces concurrently. Thus, the network is retained more load balanced which leads to improve network performance compared to the NEMO-BSP and F-NEMO.

\section{Acknowledgments}

A special thanks to the Government of Malaysia, through Ministry of Higher Education (MoHE) for the education sponsorship. We would also like to thank Research Management Center (RMC) at the International Islamic University Malaysia (IIUM) in part for the grant sponsorship.

\section{References}

[1] V. Devarapalli, R. Wakikawa, A. Petrescu and P. Thubert, "Network Mobility Basic Support Protocol (NEMO BSP)", RFC 3963, (2005).

[2] R. Kuntz, J. Montavont and T. Noel, "Multihoming in IPv6 mobile networks: progress, challenges, and solutions", Communications Magazine, IEEE, vol. 51, no. 1, (2013), pp. 128-135.

[3] X. Chen, H. Zhang, Y. Chang and H. Chao, "Experimentation, and performance analysis of multiinterfaced mobile router scheme", Simulation Modelling Practice and Theory, vol. 18, no. 4, (2010), pp. 407-415.

[4] R. Wakikawa, "Multiple care-of addresses registration", RFC 5648, (2009).

[5] G. Tsirtsis, H. Soliman, N. Montavont, G. Giaretta and K. Kuladinithi, "Flow bindings in mobile IPv6 and network mobility (NEMO) basic support", RFC 6089, (2011).

[6] C. J. Bernardos, M. Calderon and I. Soto, "PMIPv6 and Network Mobility Problem Statement Information Engineering Task Force", (2012).

[7] C. W. Lee, M. C. Chen and Y. S. Sun, "A novel network mobility management scheme supporting seamless handover for high-speed trains", Computer Communications, vol. 37, (2014), pp. 53-63.

[8] J. I. Choi, W. K. Seo and Y. Z. Cho, "Efficient network mobility support scheme for proxy mobile IPv6", EURASIP Journal on Wireless Communications and Networking, no. 1, (2015), pp. 1-13.

[9] S. Ryu, K. J. Park and J. W. Choi, "Enhanced fast handover for network mobility in intelligent transportation systems", Vehicular Technology, IEEE Transactions, vol. 63, no. 1, (2014), pp. 357-371.

[10] J. Lee and T. Ernst, "Lightweight Network Mobility within PMIPv6 for Transportation Systems", IEEE Systems Journal, vol. 5, no. 3, (2011), pp. 352-361, doi: 10.1109/JSYST.2011.2158681.5.

[11] J. Lee, N. Chilamkurti and T. Ernst, "Performance Analysis of PMIPv6-Based Network Mobility for Intelligent Transportation Systems", IEEE Transactions on Vehicular Technology, vol. 61, no. 1, (2012), pp. 74-85, doi: 10.1109/TVT.2011.2157949.

[12] G. S. Tomar and S. Verma, "Analysis of handoff Initiation using different path loss models in mobile communication system", $3^{\text {rd }}$ IEEE International conference on wireless and optical communication networks "WOCN2006", (2006), p. 5.

[13] G. S. Tomar and S. Verma, "New Call Admission Control and Handoff Techniques for 3-G Mobile Networks", IEEE International Conference on Advanced Computer Control, ICACC 2009, (2009), pp. 697-701.

\section{Authors}

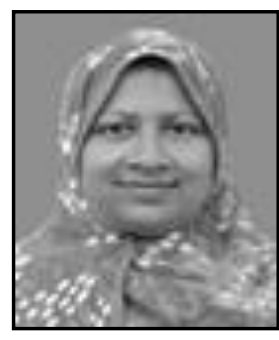

Shayla Islam, she received $\mathrm{PhD}$ in Computer and Information Engineering, at the department of Electrical and Computer Engineering in International Islamic University, Malaysia in 2016. She competed her MSc at the department of Electrical and Computer Engineering in International Islamic University, Malaysia in 2012. 
She completed her BSc in Computer Science and Engineering from International Islamic University Chittagong, Bangladesh in 2007. She was appointed as a lecturer in Green University of Bangladesh (GUB) from 2007 to 2009. Her current research interests include network mobility, computer networks and wireless communication. She published more than 45 papers in international journals and conferences. She works as a reviewer for many international conferences.

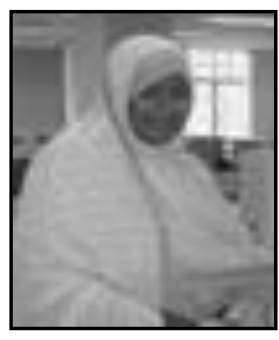

Aisha Hassan Abdalla Hashim, she received her B.Sc. and M.Sc. in Computer Engineering from University of Gezira, Sudan and her $\mathrm{PhD}$ in Computer Engineering from International Islamic University Malaysia (IIUM) in 2007. She was appointed as a lecturer in 1997. Currently she is Professor at IIUM. Her current research interests include Data Communication and Computer Networking, ASIC Design, Computer Architecture and Grid Computing, Open Sources \& Operating Systems. She published more than 100 papers in international journals and conferences. She is IEEE Senior Member, IEEE Women in Engineering Member and she works as a reviewer for many ISI journals.

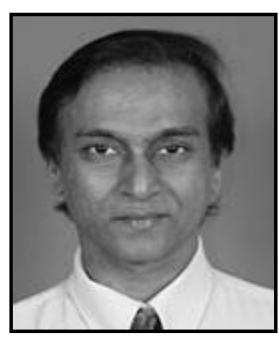

Azween Bin Abdullah, he received his bachelor degree in Computer Science from University of Southern California, USA. He then pursued his MSc in Software Engineering at Universiti Malaya, Malaysia. He completed his PhD studies from Universiti Tun Abdul Razak (UNITAR), Malaysia in the field of Information Technology (Cybersecurity). He is currently at Taylor's University, Kuala Lumpur, School of Computing and IT as a Professor. His current research interests include Software Engineering, Computer Security and Reliability, Computer Communications (Networks). Dr. Azween has published several publications on his research findings. He is a Fellow, British Computer Society - Member since 2009.

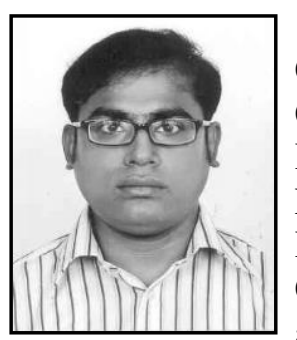

Mohammad Kamrul Hasan, he is currently a $\mathrm{PhD}$ candidate in Communication Engineering at the department of Electrical and Computer Engineering in International Islamic University, Malaysia. $\mathrm{He}$ achieved his received his Master's in Communication Engineering from International Islamic University, Malaysia in 2012. His current research interests include OFDMA, Interference, Cognitive Network, Optimization, Big Data, Smart Grid Computing, and Data Communication and Networking. He published more than 50 papers in international journals and conferences. He is a member of IEEE, MIET. 
International Journal of Future Generation Communication and Networking Vol. 9, No.8, (2016) 